\title{
Generalized $n$-Poisson brackets on a symplectic manifold
}

\author{
J. Grabowski円 \\ and \\ G. Marmo?
}

\begin{abstract}
On a symplectic manifold $(M, \omega)$ we study a family of generalized Poisson brackets associated with $2 k$-forms $\omega^{k}$. The extreme cases are related to the Hamiltonian and Liouville dynamics. We show that the Dirac brackets can be obtained in a similar way.
\end{abstract}

\section{Introduction}

Hamiltonian formalism and Poisson brackets have acquired a dominant role in the description of classical systems after their use by Dirac in the formulation of Quantum Mechanics Di1]. However, when dealing with statistical mechanics on the phase space, the Liouville measure plays a more relevant role. On a symplectic manifold (phase space), as noticed by Poincaré $[\mathrm{Pd}$, there are also available other integral invariants. To be more specific, on any $2 n$-dimensional symplectic manifold $(M, \omega)$ any Hamiltonian system in addition to $\omega$ preserves also $\omega^{2}, \omega^{3}, \ldots, \omega^{n}$. Among them $\omega$ and $\omega^{n}$ play a privileged role, because they define isomorphisms between covariant and contravariant tensors, intermediate powers do not.

Vector fields preserving a volume form are divergenceless vector fields, i.e. they represent continuity equations and therefore define 'conserved quantities'. Vector fields preserving a volume form have been called Liouville

\footnotetext{
${ }^{1}$ Institute of Mathematics, Warsaw University, ul. Banacha 2, 02-097 Warszawa, Poland; e-mail: jagrab@mimuw.edu.pl . Supported by KBN, grant No. 2 P03A 04210.

${ }^{2}$ Dipartimento di Scienze Fisiche, Università di Napoli, Mostra d'Oltremare, Pad. 20, 80125 Napoli, Italy; e-mail: gimarmo@na.infn.it .

This work has been partially supported by PRIN-97 "SINTESI".
} 
dynamical systems [MSSZ]. They furnish a geometrical approach to all dynamical systems which satisfy some continuity conditions. For these systems it is possible to study the analogue of Poisson brackets.

In this paper we would like to show that it is possible to introduce and study the analogue of Poisson brackets also for intermediate powers of $\omega$, hopefully the analysis of these situations from the dynamical point of view may bring in a finer classification of dynamical systems which goes beyond the dichotomy Hamiltonian-non-Hamiltonian dynamics.

We introduce very briefly what is the ideology in introducing brackets associated with powers of $\omega$. The main observation is that, if on a manifold $M$ of dimension $m$ we have a volume element $(m$-form) $\Omega$ and an $(m-2)$ form $\alpha$, for any two functions $f, g \in C^{\infty}(M)$ we can define the bracket $\{f, g\}$ by setting

$$
\{f, g\} \Omega=\mathrm{d} f \wedge \mathrm{d} g \wedge \alpha .
$$

Of course, we have to put additional requirements on $\alpha$, if we want to have the Jacobi identity for the bracket. On a symplectic manifold $(M, \omega)$ we recover the standard Poisson bracket if we put $\Omega=\omega^{n}$ and $\alpha=n \omega^{n-1}$.

If we use an $(m-4)$-form $\beta$, we can define a quaternary bracket by

$$
\left\{f_{1}, f_{2}, f_{3}, f_{4}\right\} \Omega=\mathrm{d} f_{1} \wedge \mathrm{d} f_{2} \wedge \mathrm{d} f_{3} \wedge \mathrm{d} f_{4} \wedge \beta
$$

and so on. When the form $\gamma$ is just a function, we get

$$
\left\{f_{1}, \ldots, f_{m}\right\} \Omega=\gamma \mathrm{d} f_{1} \wedge \cdots \wedge \mathrm{d} f_{m}
$$

or

$$
\left\{f_{1}, \ldots, f_{m}\right\}=\gamma \operatorname{det}\left(\frac{\partial f_{i}}{\partial x_{j}}\right) .
$$

which is the prototype of a Nambu bracket $\mathbb{N a}$ (cf. also GMP).

Previous idea can be used also to deal with brackets in the presence of 'second class constraints' [Di1, Di2, MMS]. If $\chi_{1}, \chi_{2}, \ldots, \chi_{2 k}$ are functions on a symplectic manifold such that

$$
\begin{gathered}
\mathrm{d} \chi_{1} \wedge \mathrm{d} \chi_{2} \wedge \cdots \wedge \mathrm{d} \chi_{2 k} \neq 0, \\
\operatorname{det}\left(\left\{\chi_{j}, \chi_{k}\right\}\right) \neq 0,
\end{gathered}
$$

then we may define the 'Dirac bracket' $\{,\}_{D}$ by

$$
\begin{gathered}
(\mathrm{d} f \wedge \mathrm{d} g) \wedge \mathrm{d} \chi_{1} \wedge \chi_{2} \wedge \cdots \wedge \mathrm{d} \chi_{2 k} \wedge \omega^{n-k-1}= \\
\{f, g\}_{D} \mathrm{~d} \chi_{1} \wedge \chi_{2} \wedge \cdots \wedge \mathrm{d} \chi_{2 k} \wedge \omega^{n-k} .
\end{gathered}
$$


Various generalizations of Poisson brackets, recently dealt within the literature, admit as a prototype those associated with powers of the symplectic structure on a symplectic manifold or a variant of it. These brackets are usually expressed in terms of multivector fields. The multivector fields giving rise to our brackets are defined by $i_{\Lambda_{\omega}} \Omega=\omega$ (see our Theorem 1 in the next section). For bivector fields we recall GMP that

$$
i_{\left[\Lambda_{1}, \Lambda_{2}\right]} \Omega=-i_{\Lambda_{1}} i_{\Lambda_{2}} \mathrm{~d} \Omega-\mathrm{d} i_{\Lambda_{2} \wedge \Lambda_{1}} \Omega+i_{\Lambda_{1}} \mathrm{~d} i_{\Lambda_{2}} \Omega+i_{\Lambda_{2}} \mathrm{~d} i_{\Lambda_{1}} \Omega .
$$

A bivector field $\Lambda$ defines a Poisson bracket if and only if

$$
\mathrm{d} i_{\Lambda \wedge \Lambda} \Omega=2 i_{\Lambda} \mathrm{d} i_{\Lambda} \Omega .
$$

In this way we can deal also with odd-dimensional manifolds (contact manifolds, for instance). The generalization, to include also Jacobi brackets, requires the introduction of brackets on modules rather than on rings of functions. A manifold $M$ which is furnished with a bi-vector field $\Lambda$ and a vector field $X$ satisfying

$$
\begin{gathered}
{[X, \Lambda]=0,} \\
{[\Lambda, \Lambda]=2 X \wedge \Lambda,}
\end{gathered}
$$

where the brackets are the Schouten brackets, is called Jacobi manifold with the Jacobi bracket DLM

$$
\{f, g\}=\Lambda(f, g)+f X(g)-g X(f) .
$$

If now, on the manifold $M \times \mathbb{R}$, we consider the bracket associated to the bivector field $e^{-2 s}\left(\Lambda+\partial_{s} \wedge X\right)$ and evaluate it on the $C^{\infty}(M)$-module of functions $\left\{\tilde{f}=e^{s} f: f \in C^{\infty}(M)\right\}$, we find that we recover the Jacobi bracket on $M$ :

$$
e^{-2 s}\left(\Lambda+\partial_{s} \wedge X\right)(\tilde{f}, \tilde{g})=\Lambda(f, g)+f X(g)-g X(f) .
$$

\section{Generalized $n$-Poisson brackets}

The following theorem describes the relation of $k$-brackets defined by differential forms (a volume $m$-form and an $(m-k)$-form) to multivector fields. 
Theorem 1 Let $\Omega$ be a volume $m$-form on a manifold $M$ and let $\alpha$ be an $(m-k)$-form. Then the $k$-bracket of functions defined by

$$
\left\{f_{1}, \ldots, f_{k}\right\} \Omega=\mathrm{d} f_{1} \wedge \cdots \wedge \mathrm{d} f_{k} \wedge \alpha
$$

is generated by the $k$-vector field $\Lambda$ defined by $i_{\Lambda} \Omega=\alpha$, i.e.

$$
\left\{f_{1}, \ldots, f_{k}\right\}=<\mathrm{d} f_{1} \wedge \cdots \wedge \mathrm{d} f_{k}, \Lambda>.
$$

Proof. The bracket satisfies clearly the Leibniz rule, so it is generated by a $k$-vector field $\Lambda$. Contractions with the volume form $\Omega$ give rise to isomorphisms between the corresponding contravariant and covariant tensors and we have just to prove that

$$
<\mathrm{d} f_{1} \wedge \cdots \wedge \mathrm{d} f_{k}, \Lambda>\Omega=\mathrm{d} f_{1} \wedge \cdots \wedge \mathrm{d} f_{k} \wedge\left(i_{\Lambda} \Omega\right) .
$$

Since it is enough to prove (2.14) pointwise, we may just work in a vector space $V$ with a basis $X_{1}, \ldots, X_{m}$ and the volume $\Omega=X_{1} \wedge \cdots \wedge X_{m}$. Let $X_{1}^{*}, \ldots, X_{m}^{*}$ be the dual basis. We have to prove that for any $I=\left(i_{1}, \ldots, i_{k}\right)$, $1 \leq i_{1}<\cdots<i_{k} \leq m$, we have

$$
<X_{i_{1}} \wedge \cdots \wedge X_{i_{k}}, \Lambda>\Omega=X_{i_{1}} \wedge \cdots \wedge X_{i_{k}} \wedge\left(i_{\Lambda} \Omega\right)
$$

for any $\Lambda \in \Lambda^{k} V^{*}$. Let us write

$$
\Lambda=\sum_{I=\left(i_{1}, \cdots, i_{k}\right)} a_{I} X_{i_{1}}^{*} \wedge \cdots \wedge X_{i_{k}}^{*} .
$$

Then

$$
i_{\Lambda} \Omega=\sum_{I=\left(i_{1}, \cdots, i_{k}\right)} a_{I} i_{X_{i_{1}}^{*} \wedge \cdots \wedge X_{i_{k}}^{*}} X_{1} \wedge \cdots \wedge X_{m}
$$

and (2.15) reduces to

$$
X_{i_{1}} \wedge \cdots \wedge X_{i_{k}} \wedge i_{X_{i_{1}}^{*} \wedge \cdots \wedge X_{i_{k}}^{*}}\left(X_{1} \wedge \cdots \wedge X_{m}\right),
$$

which is obvious. 
Lemma 1 If $\Lambda_{1}, \ldots, \Lambda_{m}$ are pairwise compatible Poisson tensors, i.e. $\left[\Lambda_{i}, \Lambda_{j}\right]=$ 0 , where [, ] is the Schouten bracket, then any wedge-product of the tensors $\Lambda_{1}, \ldots, \Lambda_{m}$ commutes with any other wedge-product of them with respect to the Schouten bracket. In particular, if $\Lambda$ is a Poisson tensor, then

$$
\left[\Lambda^{i}, \Lambda^{j}\right]=0
$$

for all $i, j=1,2, \ldots$, where

$$
\Lambda^{i}=\underbrace{\Lambda \wedge \cdots \wedge \Lambda}_{i \text {-times }}
$$

Proof. It follows immediately from the Leibniz rule for the Schouten bracket

Remark. The same remains valid for any Nambu-Poisson structure $\Lambda$ by similar arguments.

Let now $\omega$ be a symplectic form on an $2 n$-dimensional manifold $M$ and let $\Lambda$ be the corresponding Poisson tensor $\Lambda=\omega^{-1}$.

\section{Lemma 2}

$$
i_{\Lambda} \omega^{k}=k(n-k+1) \omega^{k-1} .
$$

Proof. Working in a Darboux chart, we have

$$
\begin{gathered}
i_{\Lambda} \omega^{k}=i_{\left(\sum_{j=1}^{n} \partial_{p_{j}} \wedge \partial q_{j}\right)} \omega^{k}=\sum_{j=1}^{n} i_{\partial_{q_{j}}} i_{\partial_{p_{j}}} \omega^{k}= \\
\sum_{j=1}^{n} i_{\partial_{q_{j}}}\left(k \mathrm{~d} q_{j} \wedge \omega^{k-1}\right)=k \sum_{j=1}^{n}\left(\omega^{k-1}+(k-1) \mathrm{d} q_{j} \wedge \mathrm{d} p_{j} \wedge \omega^{k-2}\right)= \\
k n \omega^{k-1}-k(k-1) \omega^{k-1}=k(n-k+1) \omega^{k-1} .
\end{gathered}
$$

Theorem 2 The $2 k$-bracket defined by

$$
\left\{f_{1}, \cdots, f_{2 k}\right\} \frac{\omega^{n}}{n !}=k ! \mathrm{d} f_{1} \wedge \cdots \wedge \mathrm{d} f_{2 k} \wedge \frac{\omega^{n-k}}{(n-k) !}
$$

is generated by the $2 k$-vector field $\Lambda^{k}$. It is an $2 k$-Poisson bracket in the sense of APP1, APP马, APP马. 
Proof. Using Lemma 2 we can prove inductively that

$$
i_{\Lambda^{k}} \frac{\omega^{n}}{n !}=\underbrace{i_{\Lambda} \cdots i_{\Lambda}}_{k-\text { times }} \frac{\omega^{n}}{n !}=k ! \frac{\omega^{n-k}}{(n-k) !}
$$

which shows, in view of Lemma 2, that the bracket is induced by $\Lambda^{k}$. According to Lemma $2, \Lambda^{k}$ is an $2 k$-Poisson structure.

Example We shall consider $M=T^{*} \mathbb{R}^{3}$ with the symplectic structure $\omega_{B}=\omega_{0}+\varepsilon_{i j k} B^{i} \mathrm{~d} q^{j} \wedge \mathrm{d} q^{k}$. It is possible to compute $\omega^{2}$ and $\omega^{3}$ and find

$$
\begin{aligned}
& \omega^{2}=\omega_{0}^{2}+\left(B^{1} \mathrm{~d} p_{1}+B^{2} \mathrm{~d} p_{2}+B^{3} \mathrm{~d} p_{3}\right) \wedge \mathrm{d} q^{1} \wedge \mathrm{d} q^{2} \wedge \mathrm{d} q^{3}, \\
& \omega^{3}=\omega_{0}^{3} .
\end{aligned}
$$

It is interesting to notice that $\omega^{3}$ does not keep track of the presence of the magnetic field. This property is sometimes quoted to account for the fact that there is no 'diamagnetism' at the classical level.

The use of $\omega^{2}$ for computing brackets in the form

$$
\left\{f_{1}, f_{2}\right\} \Omega=\mathrm{d} f_{1} \wedge \mathrm{d} f_{2} \wedge \omega^{2}
$$

will reproduce the standard bracket associated with $\omega_{B}$, however in this way of computing we show immediately that, while $\left\{q^{i}, q^{j}\right\}=0$, we find now $\left\{p_{i}, p_{j}\right\}=\varepsilon_{i j k} B^{k}$ and the Jacobi identity is equivalent to

$$
\operatorname{div} \vec{B}=\frac{\partial B^{1}}{\partial q^{1}}+\frac{\partial B^{2}}{\partial q^{2}}+\frac{\partial B^{3}}{\partial q^{3}}=0 .
$$

As for $\left\{p_{i}, q^{j}\right\}$ we see that the product

$$
\mathrm{d} p_{i} \wedge \mathrm{d} q^{j} \wedge(\vec{B} \mathrm{~d} \vec{p}) \wedge \mathrm{d} q^{1} \wedge \mathrm{d} q^{2} \wedge \mathrm{d} q^{3}=0,
$$

therefore it remains unchanged, $\left\{p_{i}, q^{j}\right\}=\delta_{i}^{j}$; it does not depend on the magnetic field.

As for quaternary bracket

$$
\left\{f_{1}, f_{2}, f_{3}, f_{4}\right\} \Omega=\mathrm{d} f_{1} \wedge \mathrm{d} f_{2} \wedge \mathrm{d} f_{3} \wedge \mathrm{d} f_{4} \wedge \omega
$$

we have

$$
X_{p_{1}, p_{2}, p_{3}}=B^{i} \frac{\partial}{\partial q^{i}},
$$


with the standard symplectic structure it would be zero. Notice that $X_{p_{1}, p_{2}, p_{3}}$ is not an inner derivation. This is a peculiar aspect of the brackets associated with intermediate powers of $\omega$, from $\omega^{2}$ to $\omega^{n-1}$, i.e. their 'hamiltonian vector fields' do not preserve the bracket.

As a further example of brackets associated with powers of $\omega$ we consider an action of a Lie group $G$ on $T^{*} \mathbb{R}^{3}$. If $G$ is any simple 3-dimensional Lie group acting on $\mathbb{R}^{3}$ with the corresponding canonical action on $T^{*} \mathbb{R}^{3}$ and the associated momentum map $J: T^{*} \mathbb{R}^{3} \rightarrow \mathfrak{g}^{*}$, we find that $X_{J_{1}, J_{2}, J_{3}}$ corresponds to the vector field associated with the Casimir function on $\mathfrak{g}^{*}$ and, moreover, is an inner derivation of the quaternary bracket. In general we find

$$
X_{f_{1}, f_{2}, f_{3}}=\left\{f_{1}, f_{2}\right\} X_{f_{3}}+\left\{f_{2}, f_{3}\right\} X_{f_{1}}+\left\{f_{3}, f_{1}\right\} X_{f_{2}}
$$

which explains why vector fields associated with three functions are not inner derivations. Also for 3-dimensional simple Lie algebras we have $C=J_{1}^{2} \pm$ $J_{2}^{2} \pm J_{3}^{2}$ with $\left\{J_{i}, J_{k}\right\}= \pm \varepsilon_{i k j} J_{j}$.

\section{Dirac brackets}

Let us assume that on a symplectic manifold $(M, \omega)$ we have functions $\chi_{1}, \ldots, \chi_{2 k}$ such that

$$
\begin{gathered}
\mathrm{d} \chi_{1} \wedge \mathrm{d} \chi_{2} \wedge \cdots \wedge \mathrm{d} \chi_{2 k} \neq 0 \\
\operatorname{det}\left(\left\{\chi_{j}, \chi_{k}\right\}\right) \neq 0 .
\end{gathered}
$$

Dirac introduced a new Poisson bracket $\{,\}_{D}$ by putting

$$
\{f, g\}_{D}=\{f, g\}-\left\{f, \chi_{i}\right\} c_{i j}\left\{\chi_{j}, g\right\}
$$

where $\left(c_{i j}\right)$ is the inverse of the matrix $\left(\left\{\chi_{i}, \chi_{j}\right\}\right)$. It is easy to see that $\chi_{i}$ are Casimir functions with respect to this new bracket. We have the following.

Theorem 3 The Dirac bracket $\{,\}_{D}$ is defined by the equation

$$
\begin{gathered}
(\mathrm{d} f \wedge \mathrm{d} g) \wedge \mathrm{d} \chi_{1} \wedge \mathrm{d} \chi_{2} \wedge \cdots \wedge \mathrm{d} \chi_{2 k} \wedge \omega^{n-k-1}= \\
\{f, g\}_{D} \mathrm{~d} \chi_{1} \wedge \mathrm{d} \chi_{2} \wedge \cdots \wedge \mathrm{d} \chi_{2 k} \wedge \omega^{n-k} .
\end{gathered}
$$


Proof. Denote by $X_{f}\left(\right.$ resp. $\left.X_{f}^{D}\right)$ the hamiltonian vector field with the hamiltonian function $f$ with respect to the original Poisson bracket (resp. Dirac bracket). Since $\chi_{i}$ 's are Casimir functions with respect to the Dirac bracket we have $X_{f}^{D}\left(\chi_{i}\right)=0$ for $i=1, \ldots, 2 k$ and all possible $f$. In particular,

$$
i_{X_{f}^{D}}\left(\mathrm{~d} \chi_{1} \wedge \cdots \wedge \mathrm{d} \chi_{2 k}\right)=0 .
$$

Moreover,

$$
\begin{array}{r}
i_{X_{f}^{D}} \omega=i_{X_{f}} \omega-\left\{f, \chi_{i}\right\} c_{i j} i_{X_{\chi_{j}}} \omega= \\
-\mathrm{d} f+\left\{f, \chi_{i}\right\} c_{i j} \mathrm{~d} \chi_{j},
\end{array}
$$

so that

$$
\mathcal{L}_{X_{f}^{D}} \omega=\mathrm{d}\left(i_{X_{f}^{D}} \omega\right)=\mathrm{d}\left(\left\{f, \chi_{i}\right\} c_{i j}\right) \wedge \mathrm{d} \chi_{j} .
$$

Hence,

$$
\begin{gathered}
\mathcal{L}_{X_{f}^{D}}\left(\mathrm{~d} \chi_{1} \wedge \cdots \wedge \mathrm{d} \chi_{2 k} \wedge \omega^{n-k}\right)= \\
\mathcal{L}_{X_{f}^{D}}\left(\mathrm{~d} \chi_{1} \wedge \cdots \wedge \mathrm{d} \chi_{2 k}\right) \wedge \omega^{n-k}+ \\
(n-k) \mathrm{d} \chi_{1} \wedge \cdots \wedge \mathrm{d} \chi_{2 k} \wedge \mathcal{L}_{X_{f}^{D}} \omega \wedge \omega^{n-k-1}=0 .
\end{gathered}
$$

Now, we can write

$$
\begin{gathered}
\{f, g\}_{D} \mathrm{~d} \chi_{1} \wedge \cdots \wedge \mathrm{d} \chi_{2 k} \wedge \omega^{n-k}= \\
\mathcal{L}_{X_{f}^{D}}\left(g \mathrm{~d} \chi_{1} \wedge \cdots \wedge \mathrm{d} \chi_{2 k} \wedge \omega^{n-k}\right)= \\
\mathrm{d}\left(i_{X_{f}^{D}}\left(g \mathrm{~d} \chi_{1} \wedge \cdots \wedge \mathrm{d} \chi_{2 k} \wedge \omega^{n-k}\right)\right)= \\
(n-k) \mathrm{d}\left(g \mathrm{~d} \chi_{1} \wedge \cdots \wedge \mathrm{d} \chi_{2 k} \wedge\left(i X_{f}^{D} \omega\right) \wedge \omega^{n-k-1}\right)= \\
-(n-k) \mathrm{d}\left(g \mathrm{~d} f \wedge \mathrm{d} \chi_{1} \wedge \cdots \wedge \mathrm{d} \chi_{2 k} \wedge \omega^{n-k-1}\right)= \\
(n-k) \mathrm{d} f \wedge \mathrm{d} g \wedge \mathrm{d} \chi_{1} \wedge \cdots \wedge \mathrm{d} \chi_{2 k} \wedge \omega^{n-k-1} .
\end{gathered}
$$

\section{Generalization of previous brackets}

This construction can be generalized by replacing exterior products of $\omega$ with multivector fields of even order on manifolds of even or odd dimensions. Now 
we have to require the vanishing of the Schouten brackets because this will not follow automatically. Therefore we can apply our procedure to general manifolds and general multivectors (see [LLMD.

At the moment it is not easy to exhibit applications of these brackets to interesting physical systems. Definitely, we could use them to select dynamical systems (vector fields) on the carrier space $M$ either by requiring the fields to be derivations of the brackets or by associating vector fields with $k$-ples of functions. This would allow for a classification that goes beyond Hamiltonian or non-Hamiltonian dynamics.

We would like to comment also that these brackets arising on a symplectic manifold are all 'natural' in the given context. The generalization to arbitrary manifolds and arbitrary multivectors will lose many of the properties that we encounter on a symplectic manifold, nevertheless they are worth investigating if one keeps in mind possible applications for dynamical systems.

\section{References}

[AM] Abraham, R.; Marsden, J .E., Foundations of Mechanics, Benjamin/Cummings 1978.

[APP1] Azcárraga, J. A.; Perelomov, A. M.; Pérez Bueno, J. C., New generalized Poisson structures, J. Phys. A: Math. Gen. 29 (1996), 627-649.

[APP2] Azcárraga, J. A.; Perelomov, A. M.; Pérez Bueno, J. C., The Schouten-Nijenhuis brackets, cohomology, and generalized Poisson structures, J. Phys. A: Math. Gen. 29 (1996), 7993-8009.

[APP3] Azcárraga, J. A.; Perelomov, A. M.; Pérez Bueno, J. C., New generalized Poisson structures, J. Phys. A: Math. Gen. 29 (1996), L151L157.

[DLM] Dazord, P.; Lichnerowicz, A.; Marle, Ch.M., Structure locale des varietes de Jacobi, J. Math. pures et Appl. 70 (1995), 101-152.

[Di1] Dirac, P.A.M., Lectures on Quantum Mechanics, Belfer Graduate School of Science, Yeshiva Univ. N.Y. 1954.

[Di2] Dirac, P.A.M., The Principles of Quantum Mechanics, 4th ed., Oxford Univ. Press, Oxford 1958. 
[Ga] Gautheron, P., Some remarks concerning Nambu mechanics, Lett. Math. Phys. 37 (1996), 103-116.

[GMP] Grabowski, J.; Marmo, G.; Perelomov, A. M., Poisson structures: Towards a classification, Modern Phys. Lett. A 8 (1993), 1719-1733.

[ILMD] Ibáñez, R.; de Leon, M.; Marrero, J. C.; de Diego, M. D., Dynamics of generalized Poisson and Nambu-Poisson brackets, J. Math. Phys. 38 (1997), 2332-2344.

[MMS] Marmo, G.; Mukunda, N.; Samuel, J., Dynamics and symmetry for constrained systems: a geometrical analysis, La Revista del Nuovo Cimento 6 (1983), 1-62.

[MSSZ] Marmo, G.; Saletan, E.J.; Simoni, A.; Zaccaria, F., Liouville dynamics and Poisson brackets, J. Math. Phys. 19 (1978), 1156.

[Na] Nambu, Y., Generalized Hamiltonian mechanics, Phys. Rev. D7 (1973), 2405-2412.

[Po] Poincarè, H., Le méthodes nouvelles de la Méchanique celesta, Vol. III, Dover, New York, 1957.

[Ta] Takhtajan, L., On foundation of the generalized Nambu mechanics, Commun. Math. Phys. 160 (1994), 295-315. 